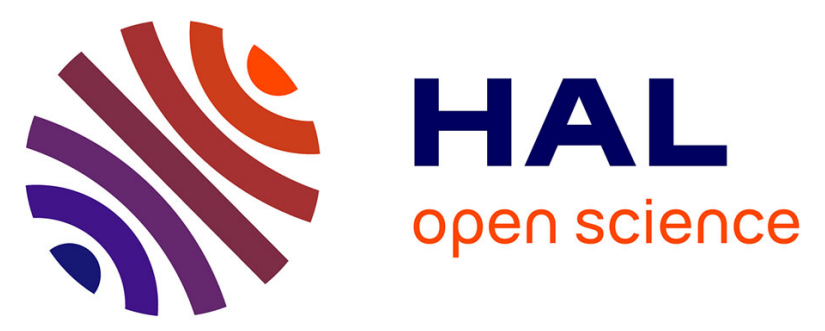

\title{
Sustainable Traffic Aware Duty-Cycle Adaptation in Harvested Multi-Hop Wireless Sensor Networks
}

Gabriele Romaniello, Olivier Alphand, Roberto Guizzetti, Andrzej Duda

\section{To cite this version:}

Gabriele Romaniello, Olivier Alphand, Roberto Guizzetti, Andrzej Duda. Sustainable Traffic Aware Duty-Cycle Adaptation in Harvested Multi-Hop Wireless Sensor Networks. Proc. the IEEE 81st Vehicular Technology Conference (VTC2015-Spring), IEEE, May 2015, Glasgow, United Kingdom. 10.1109/VTCSpring.2015.7146117 . hal-01287516

\section{HAL Id: hal-01287516 https://hal.science/hal-01287516}

Submitted on 13 Mar 2016

HAL is a multi-disciplinary open access archive for the deposit and dissemination of scientific research documents, whether they are published or not. The documents may come from teaching and research institutions in France or abroad, or from public or private research centers.
L'archive ouverte pluridisciplinaire HAL, est destinée au dépôt et à la diffusion de documents scientifiques de niveau recherche, publiés ou non, émanant des établissements d'enseignement et de recherche français ou étrangers, des laboratoires publics ou privés. 


\title{
Sustainable Traffic Aware Duty-Cycle Adaptation in Harvested Multi-Hop Wireless Sensor Networks
}

\author{
Gabriele Romaniello* ${ }^{\text {\} } \text {, Olivier Alphand*, Roberto Guizzetti }{ }^{\llbracket} \text {, and Andrzej Duda* }$ \\ ${ }^{*}$ Grenoble Institute of Technology, CNRS Grenoble Informatics Laboratory UMR 5217, France \\ ISTMicroelectronics, Crolles, France \\ Email: \{firstname.lastname\}@imag.fr, \{firstname.lastname\}@ st.com
}

\begin{abstract}
Sustainable power management techniques in energy harvesting wireless sensors currently adapt the consumption of sensors to their harvesting rate within the limits of their battery residual energy, but regardless of the traffic profile. To provide a fairer distribution of the energy according to application needs, we propose a new sustainable traffic aware duty-cycle adaptation scheme (STADA) that takes into account the traffic load in addition to previous factors. We evaluate our protocol in the specific context of multi-hop IEEE 802.15.4 beacon-enabled wireless sensor networks powered by solar energy. Simulations show that our solution outperforms traffic-unaware adaptation schemes while minimizing the variance of the quality of service provided to applications.
\end{abstract}

\section{INTRODUCTION}

Wireless Sensor Networks powered by ambient energy harvesting (WSN-HEAP) may substantially extend their lifetime by taking advantage of ambient sources of energy to recharge the sensor battery. If nodes achieve a proper energy balance between all energy consuming activities and the energy harvesting intake, the network could even last for ever.

To perform energy balance, advanced consumption adaptation schemes currently rely on the energy harvesting rate and the battery residual energy regardless of the traffic load. Therefore, the supplied energy is not always consistent with the traffic profile leading to an irregular quality of service and a non-optimal use of energy. For instance, a high duty-cycle when traffic is low does not bring much in terms of throughput, but leads to a waste of energy that could have been better used later in case of a traffic increase or a decreasing harvesting rate.

Moreover, we have decided to focus on indoor scenarios and choose light (natural and artificial) as the ambient source of energy, because it is abundant, easy to access, and benefits from a proper power density. Unlike solar profiles which diurnal and seasonal variations can be predicted, indoor light profiles may be quite unpredictable because of the fluctuation of its artificial component. Therefore, we favor a predictionless approach to estimate the energy harvesting rate.

At last, we propose to address the adaptation issue in a specific highly constrained context of multi-hop 802.15.4 networks operating in the beacon-enabled mode. Maintaining energy balance while still meeting good network performance is difficult, because harvested energy and traffic load are unevenly distributed in space and in time. Thus, nodes may obtain different solar energy at different places and, for instance in a convergecast scenario, the traffic load is heavier for the nodes closer to the sink. An additional difficulty is that, when each node independently adapts its duty-cycles to local conditions, it may influence the operation of other nodes on the path to the sink.

For all these reasons, we propose a new sustainable traffic aware duty-cycle adaptation algorithm (STADA) that fits the constraints of multi-hop energy harvesting wireless sensor networks. It takes into account the traffic load in addition to the harvesting rate and the battery level. We perform extensive simulations to analyze the performance of our algorithm in terms of delay and packet delivery ratio as well as a fairer distribution of energy in function of the traffic needs. We also compare our solution to a traffic unaware algorithm [1].

The rest of the paper is organized as follows. Section II recalls the principles of the 802.15 .4 operation and energy harvesting. Section III presents the proposed adaptation algorithm and Section V reports simulation results. Finally, Section VI discusses the related work and Section VII concludes the paper.

\section{BACKGROUND}

\section{A. IEEE 802.15.4 Networks}

The IEEE 802.15.4 standard for Low-Rate Wireless Personal Area Networks (LR-WPANs) [2] defines the physical and the MAC (Medium Access Control) layers and two operating modes. In the beacon-enabled mode, a coordinator node periodically sends a beacon to delimit its superframes and invites neighboring associated nodes to send their frames during the Contention Access Period (CAP). To avoid collisions during the CAP, all children use the slotted CSMA-CA method to access the medium.

In the beacon-enabled mode, two parameters determine the duty-cycle: Beacon Order (BO) and Superframe Order (SO) with integer values satisfying $0 \leq \mathrm{SO} \leq \mathrm{BO} \leq 14$. They define the time between two successive beacons (BI, Beacon Interval):

$$
B I=\text { aBaseSuperframeDuration } \cdot 2^{B O}
$$

and the duration of the active phase (SD, Superframe Duration):

$$
S D=\text { aBaseSuperframeDuration } \cdot 2^{S O} .
$$




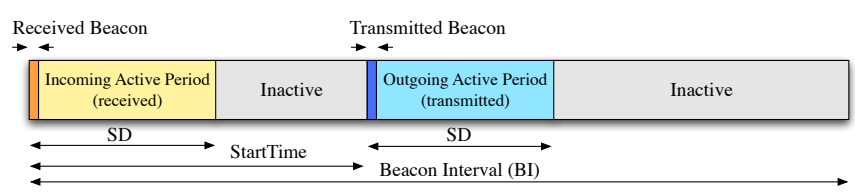

Fig. 1: Outgoing and incoming superframes

To support multi-hop topologies (i.e. cluster-trees), the 802.15.4 working group defined an Outgoing Superframe $\left(S F_{\text {out }}\right)$ dedicated to communications of a node with its child nodes and an Incoming Superframe $\left(S F_{\text {inc. }}\right.$ ) for communications with its parent node interspaced by STARTTIME (cf. Fig. 1). Nodes may sleep during the inactive parts of the superframe. Nodes may additionally sleep during $S F_{\text {inc }}$. if they have no traffic to send to their coordinator. On the contrary, during $S F_{\text {out }}$, nodes have to listen during the whole superframe to possible transmissions coming from their sons. A particular care has to be taken for the scheduling of superframes among the coordinators in the two-hop neighborhood, because they may overlap and in this case, beacons and frames may collide. We use a static multichannel allocation scheme of superframes over 16 channels to avoid beacon collisions.

Another limitation of the standard is that $S F_{\text {out }}$. and $S F_{\text {inc. }}$. share the same duration. Therefore, the duty-cycle of all coordinators belonging to the same Personal Area Network (PAN) is fixed (in time) and homogeneous (in space). However, in our multi-hop harvesting context, nodes do not necessarily face the same traffic and light conditions. Thus, each coordinator should be able to dynamically adapt its own duty-cycle to its local light conditions and traffic load.

\section{B. Energy Harvesting}

Regarding harvesting, we consider sensors that convert light energy to electrical energy through small solar panels of $5 \times 4 \mathrm{~cm}$. Their efficiency is around $3-5 \%$ and the maximum power they generate is $3.8 \mathrm{~mW}$ if we refer to both indoor light profiles we consider in Section V. Even at this power, the solar panel does not generate enough power to cope with the instantaneous consumption of a sensor node. Consequently, the energy coming from the panel is first used to recharge the battery and then the sensor draws power from the battery as soon as a minimum level is reached. Finally, we assume an ideal non-leaking battery that supports a (virtually) unlimited number of recharge cycles and therefore does not suffer from degradation issues like the reduction of storage capabilities. Thanks to those assumptions, the battery can be easily included in the model that we use to analyze the harvesting nodes detailed in Section III.

\section{HaRvesting System Model}

The model depicted in Figure 2 gives an overview of our harvesting model and the different constraints taken into account by our duty-cycle adaptation scheme.

First, we propose to model the couple battery-solar panel as a modified token-bucket that we call an energy-bucket (cf.

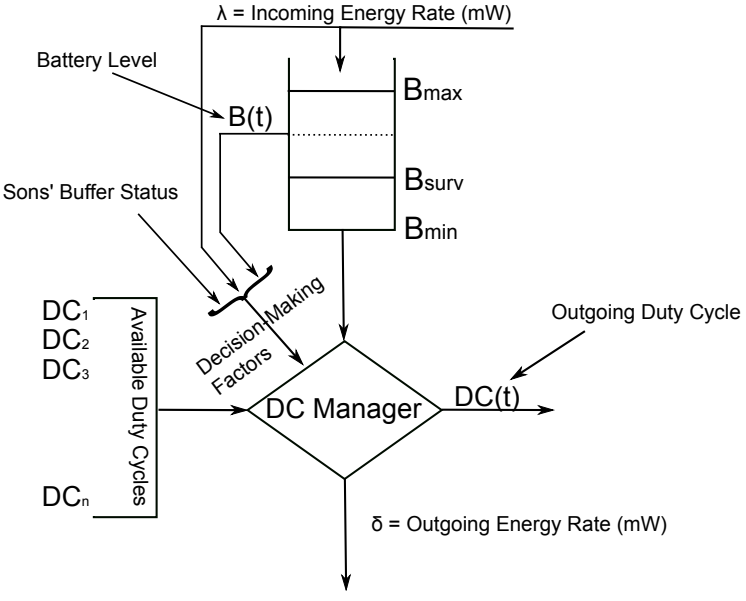

Fig. 2: Energy bucket model

Figure 2). The solar panel operation is represented by the recharging rate $\lambda$, the intake power. The energy consumption is characterized by $\delta$ (i.e Outgoing Power), the average energy consumption rate that relies on the decision of the Duty-cycle Manager detailed later in Section IV. Thus, the instantaneous battery level $B(t)$ directly depends on $\lambda$ and $\delta$. At last, we define 3 specific battery levels : $B_{\max }, B_{\min }$ and $B_{\text {surv }}$. $B_{\max }$ and $B_{\text {min }}$ represent the maximum and minimum energy values storable in the battery. If $B(t)>B_{\max }$ (i.e. the battery is full), the energy coming from the solar panel is discarded. If $B(t)<=B_{\min }$, the sensor stops working and needs to be recharged and rebooted manually. $B_{\text {surv }}$ is the minimum level of battery required to survive at an a priori fixed low dutycycle during long predefined dark periods based on predictable light profiles (nights) or computed from previous observations.

The last element is the duty-cycle manager in charge of maintaining energy balance and leading to an unlimited lifetime. In other words, it has to adapt the outgoing dutycycle so that the equation 3 is always satisfied.

$$
\int_{0}^{T} \delta(t) \leq B_{0}+\int_{0}^{T} \lambda(t) \quad \forall \quad T \in[0, \infty),
$$

\section{DUTY-CYCLE MANAGER}

The main issue our new duty-cycle adaptation scheme addresses is the waste of energy resulting from an adaptation of duty-cycle not taking into account dynamic application needs. For instance, allocating high duty-cycles when there is no traffic is counter-productive since this energy could be better used later. Similarly, decreasing the duty-cycle when the traffic is moderate is not always the best option as local contention will rise leading to expensive retransmissions and significant performance degradations.

For better understanding, we give an overview in Figure 2 of the set of parameters taken into account by the duty-cycle manager and we detail them subsequently.

We first introduce a division of time into 5-minute slices (e.g. $n=288$ per day) and assume that during a slice, a node will harvest a similar amount of energy to the previous 
slice, because of slow light variations. Let $E_{n}$ be the energy to allocate for the slice $n, H_{n}$ the energy harvested during slice $n$ (dereived from the light sensor and the battery charge), $L_{B} \in$ $(0,1)$ the proportion of the battery level, and $L_{T} \in(0,1)$ the proportion of the traffic level measured in function of the number of packets in the buffers of nodes associated with the coordinator ${ }^{1}$.

At the end of each slice, the duty-cycle manager computes the energy to allocate for the next slice as shown in Eq. 4.

$$
E_{n}=\beta \cdot H_{n-1}+\gamma \cdot H_{\max } \cdot L_{B}+\delta \cdot H_{\max } \cdot L_{T},
$$

where $\beta, \gamma, \delta$ are respectively, the weight factor of each component that satisfy the following condition $\beta+\gamma+\delta=1$ except when the battery is full (in this case, $\beta$ is set to 1 to completely consume the scavenged energy). $H_{\max }$ is the maximum energy harvested during a slice $\left(H_{\max }=1.08 \mathrm{~J}\right.$ on our platform). $H_{\max }$ is updated using an Exponentially Weighted Moving Average carried out over past days.

The duty-cycle manager provides the outgoing duty-cycle that defines the length and frequency of $S F_{\text {out }}$. for the next slice. To obtain it, we first derive the duty-cycle for the next slice according to the following formula:

$$
D C_{n}=\left(E_{n}-E_{p_{n}}\right) / E_{0},
$$

where $E_{0}$ is the energy consumed during a slice by an alwayson node and $E_{p_{n}}$ represents the energy consumed during the incoming active period. $E_{p_{n}}$ is updated according to the expression:

$$
E_{p_{n+1}}=\alpha \cdot\left[E_{p_{n}}+(1-\alpha) \cdot E_{p_{n-1}}+(1-\alpha)^{2} \cdot E_{p_{n-2}}\right]
$$

where $E_{p_{0}}$ is 0 .

Then we transform $D C_{n}$ into a combination of BO and SO that results in $D C_{n}$ with Algorithm 1.

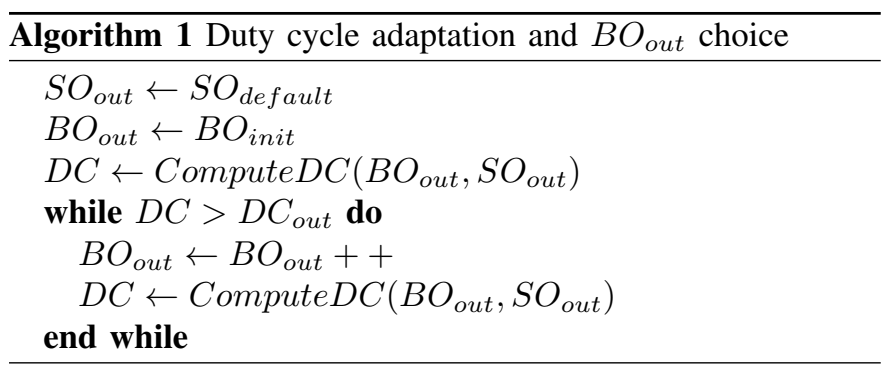

For the sake of simplicity, we have decided to fix $S O$. First, the algorithm fixes $S O_{\text {out }}$ to the default value and adjusts $B O_{\text {out }}$ starting from an initial low value. In the while loop, the node increases $\mathrm{BO}_{\text {out }}$ until the resulting duty-cycle is less than the outgoing duty-cycle. In IEEE 802.15.4 beacon-enabled mode, several combinations of $B O$ and $S O$ correspond to the same duty-cycle. We privilege the smaller values of Bo that result in shorter delays.

\footnotetext{
${ }^{1}$ To provide traffic status, additional information on the queuing occupancy of son nodes are sent in the reserved field of the MAC header ( 3 bits). The algorithm just considers the worse queuing occupancy among the sons.
}

If $L_{B} \leq L_{B}^{\min }$ (the proportion of the battery level at $\left.B_{\text {surv }}\right)$, the node automatically adopts the night configuration for $B O$ and $S O$ ( $B O_{\text {survive }}$ and $\left.S O_{\text {survive }}\right)$ for its outgoing superframe. $B O_{\text {survive }}$ and $S O_{\text {survive }}$ represent the minimum level of required quality of service.

\section{Performance eValuation}

We used the WsNet simulator [3] designed for Wireless Sensor Networks. We have integrated the accurate beacon-enabled implementation of IEEE 802.15.4 [4] that supports multihop topologies, multichannel superframe scheduling, and single duty-cycle networks. We have implemented the proposed dutycycle adaptation algorithm and extended the module to support heterogeneous duty-cycles. We developed a module for a rechargeable battery and a module that simulates the solar panel.

We have set up all simulation parameters of energy consumption and scavenging behavior to the values corresponding to STM GreenNet tag, the new generation of harvesting sensor nodes manufactured by STMicroelectronics (STM). A GreenNet tag is composed of a 32 bit microcontroller, a rechargeable battery, a 802.15 .4 beacon-enabled radio, a solar panel $(\sim 4 \times 5 \mathrm{~cm})$, and the Contiki network stack. Table I shows the STM node power consumption in each operational state.

TABLE I: Typical Operating Conditions of STM nodes.

\begin{tabular}{lrrr}
\hline Operational state 1: MCU On, Radio Rx & 30 & $\mathrm{~mW}$ \\
\hline Operational state 2: MCU On, Radio Tx & 30 & $\mathrm{~mW}$ \\
\hline Operational state 3: MCU Off, Radio Off & 8.4 & $\mu \mathrm{W}$ \\
\hline
\end{tabular}

We simulate the topology shown in Figure 3, a cluster-tree with maximal depth 4 and 23 nodes representing a Home Automation or Office Automation scenario.

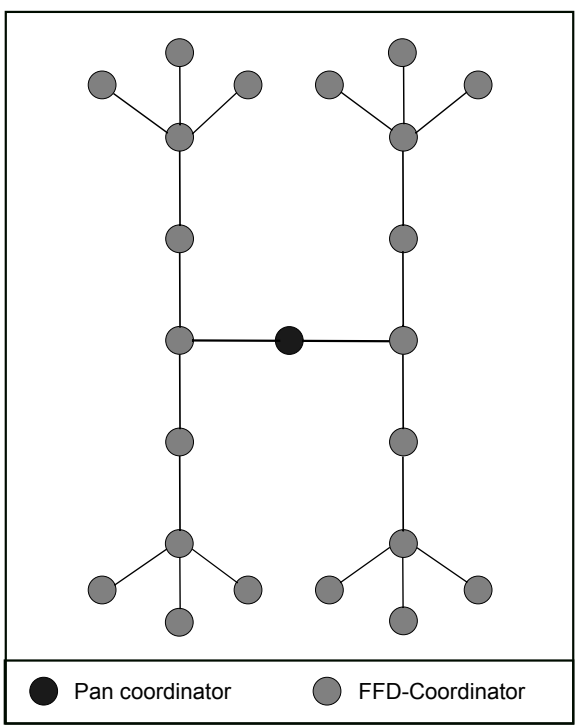

Fig. 3: Example topology

The sink (Edge Router) is main powered and uses a fixed duty-cycle $(\mathrm{BO}=4$ and $\mathrm{SO}=1)$. We use all of the 16 available 
channels provided by standard in order to avoid active period overlapping during dynamic changes of duty-cycles.

Once the battery level is below $L_{B}^{\min }$, we fix the surviving outgoing duty-cycle $\left(S F_{\text {out }}\right.$. $)$ to $\mathrm{BO}_{\text {survive }}=9$ and $\mathrm{SO}_{\text {survive }}=1$. This duty-cycle is also the minimum dutycycle that can be chosen by the duty-cycle Manager.

We fix $B O_{\text {Init }}=4$ and $S O_{\text {default }}=1$ to speed up the network bootstrap at the beginning of the simulation. This duty-cycle also provides a satisfactory quality of service for a typical traffic data rate in Home or Office Automation.

We fix the duty-cycle manager parameter with $\beta=0.5, \gamma=$ 0.25 , and $\delta=0.25$, because in our case, we want an algorithm that principally makes its decision based on the harvesting rate.

Table II summarizes all the parameters of the simulations.

TABLE II: Simulation Parameters.

\begin{tabular}{lr}
\hline Starting BO & 4 \\
\hline Starting SO & 1 \\
\hline Number of nodes & 23 \\
\hline Traffic rate & 10 up to 21 bytes per second \\
\hline Traffic type & CBR converge-cast toward sink \\
\hline Data packet size & 127 bytes \\
\hline Radio transmission range & $50 \mathrm{~m}$ \\
\hline Radio sensing range & $90 \mathrm{~m}$ \\
\hline Shadowing Model & Log-normal \\
\hline$\beta$ (weight for energy harvesting rate) & 0.5 \\
\hline$\alpha$ (weight for residual battery level) & 0.25 \\
\hline$\gamma$ (weight for sons' traffic load) & 0.25 \\
\hline
\end{tabular}

In all simulations each data point is derived from the average of five 72-hour simulations each using different seeds.

We consider two light profiles: a predictable profile and an unpredictable light.

The predictable light profile is shown in Figure 4. It is the power profile of three regular sunny days with the maximum around midday with $3.8 \mathrm{~mW}$.

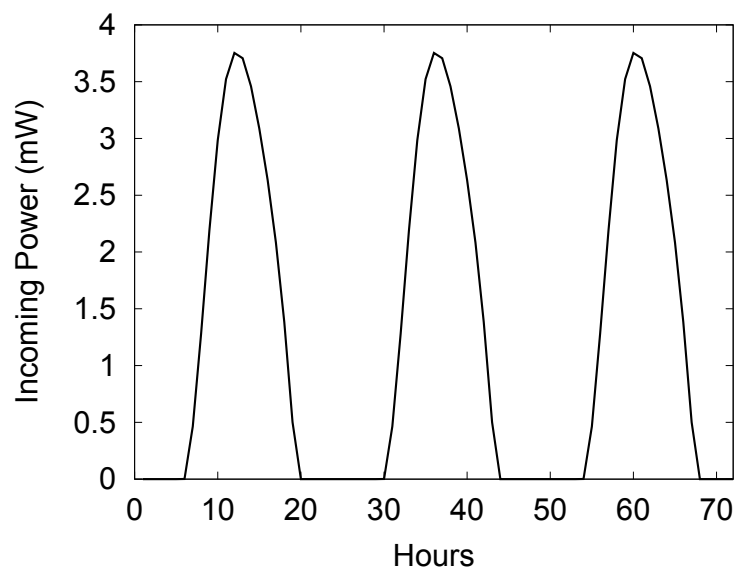

Fig. 4: Incoming power during three sunny days.

We have compared the result of STADA with Duty-cycle Scheduling based on Prospective increase in residual energy (DSP) [1].
In DSP, sensor nodes adjust their duty-cycle proportionally to the residual energy in the battery and the prospective increase (or decrease) linked to their energy harvesting rate. DSP is designed for the asynchronous RI-MAC access protocol [5] so we have to adapt it to beacon-enabled 802.15.4 networks to be able to compare it with STADA.

To exhibit the substantial differences between the two algorithms, Figure 5 shows the packet delay at the second hop in the topology shown in Figure 3.

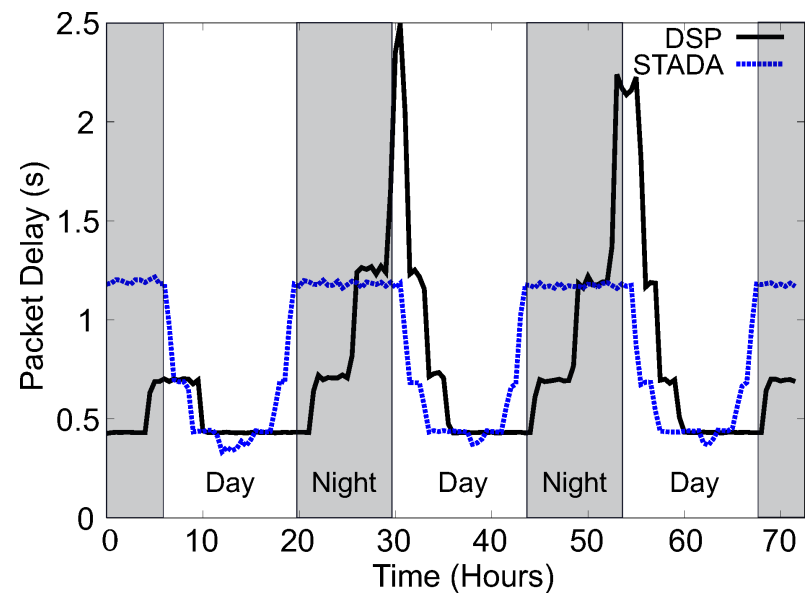

Fig. 5: Packet delay during three sunny days.

DSP in the first 10 hours is in the warm up phase. It provides high duty-cycles even without intake energy because in the beginning the battery level is high. We can notice that packets experience a larger delay and jitter with DSR than with STADA during the night period. On one hand, by reacting immediately to the change of light, STADA is able to spare energy at the beginning of the night and to redistribute it more equally over the whole night duration. On the other hand, DSP is not able to take into account the harvesting rate, so it continues to make its decision based on the battery level. The consequence is that it wastes energy in the first night phase. Then, the battery level rapidly decreases and the algorithm is forced to schedule larger BO leading to higher delays.

Figure 6 shows the cumulative energy consumptions of both STADA and DSP against the cumulative harvested energy. The recharged energy starts from $100 \mathrm{~J}$, which is the initial battery level. The distance between the harvested energy curve and the energy consumed one represents the instantaneous battery level. The shaded regions correspond to the period when there is no light (also indicated by a plateau in the cumulative harvested energy curve).

We can notice again that the energy consumption of STADA better follows the harvested energy curve. Moreover, its consumption curve is always below the DSP curve, which leads to higher battery levels during the whole simulation for STADA. Thus, for a similar (or even better) quality of service as explained later, STADA consumes less energy than DSP. Another interesting consequence is that the battery lifetime is correlated with the depth of discharge [6]. In other words, 
STADA may safeguard more recharge cycles than the DSP algorithm.

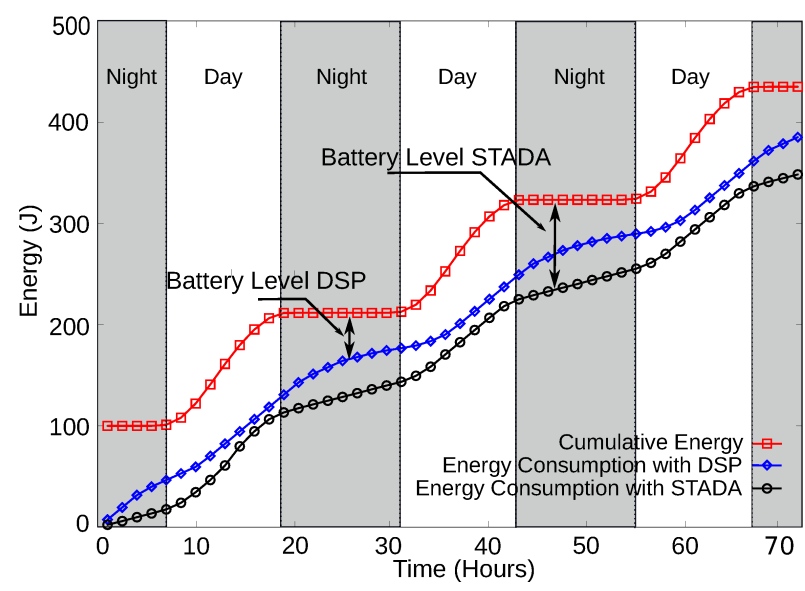

Fig. 6: Energy profile

Figure 7 shows the Cumulative Distribution Function for the delay of all packets for each algorithm. We have simulated the network with a traffic pattern set to one packet every 6 and every 12 seconds. In either cases, STADA provides better performance, even if in the configuration with the traffic set to a packet every 12 seconds the profiles are quite similar. By increasing the data rate up to one packet every 6 seconds, the difference is even more salient and packets experience shorter delays with STADA during the whole simulation as well as a better PDR.

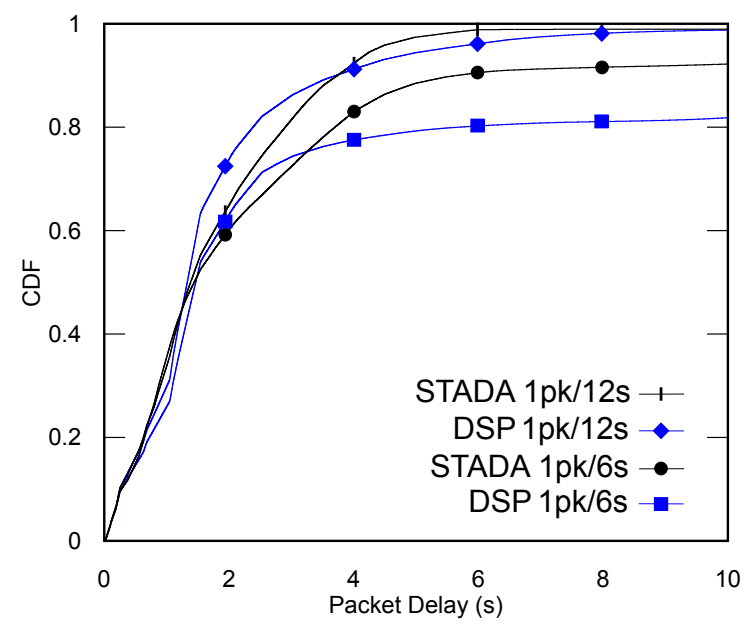

Fig. 7: CDF of packet delay during three sunny days.

We reproduce the same experiments with a non predictable profile as shown in Figure 8, which is an interpolation of real traces [7].

Figure 9 shows the Cumulative Distribution Function of the simulation with an unpredictable profile. STADA also provides better performance under both traffic patterns in terms of delay and packet delivery ratio. By taking taking into account the

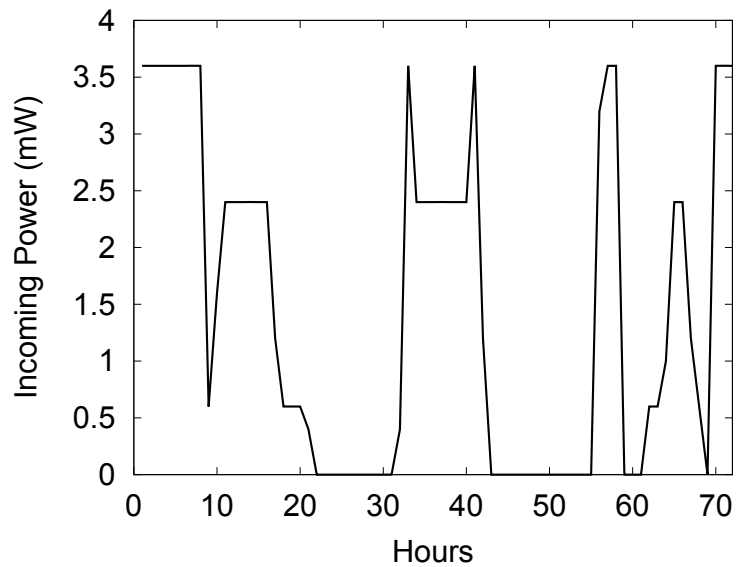

Fig. 8: Incoming power during three unpredictable days.

buffer size of son nodes, STADA is able to increase the dutycycle to avoid buffer queueing and packet drop as well as local contention that would lead to unnecessary retransmissions.

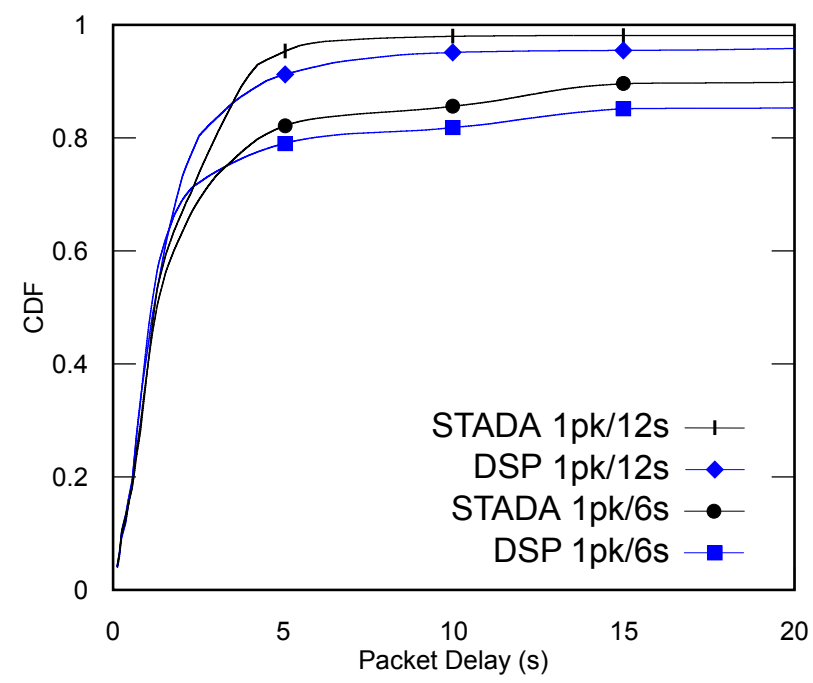

Fig. 9: CDF of packet delay for the unpredictable profile.

\section{RELATED WORK}

\section{A. Duty-cycle protocols for beacon-enabled 802.15.4}

Several authors proposed algorithms to adapt the duty-cycle to traffic in 802.15 .4 beacon-enabled, battery powered WSN but the most advanced one is DCLA [8]. It is an efficient learning algorithm that jointly changes BO and SO according to an advanced traffic estimation based on the superframe utilization (observed by the coordinator), buffer occupancy, and queuing delays (transmitted to the coordinator by each node). An exhaustive comparison with other solutions is done and DCLA clearly outperforms them under various traffic patterns.

Nevertheless, all the proposals share the same drawback: they only consider star topologies. duty-cycle adaptation in 
multi-hop topologies requires taking into account additional parameters: heterogeneous duty-cycles, the additional queued traffic that a coordinator needs to relay to its parent, or the superframe allocation conflicts.

Distributed Duty Cycle Management (DDCM) [9] tackles those issues but it relies on the new 802.15.4e standard [10] which supports mesh topologies as well as the allocation/deallocation of extra superframes (in addition to the traditional outgoing superframe), which provides a finer granularity and a higher flexibility not possible in the original 802.15.4.

\section{B. Duty-cycling in sustainable wireless sensor networks}

Several research papers are published to make the WSNHEAPs auto-sustainable and increase their performances. Kansal et al. [11] dynamically adapt the duty-cycle according to the deviations from the expected energy input learned from historical observations. They assume a periodic and predictable light profile and use a prediction mechanism in order to estimate the future energy supply. Prediction tools suffer of prediction errors and require a non negligible storage for low power sensor nodes. Vigorito et al. proposed an alternative model-free approach to solve the duty-cycle problem in the HEAP context (energy harvesting) using techniques from adaptive control theory [12]. The proposed solution does not make any assumption about the nature and dynamics of the energy source, making their approach more easily implemented in real systems. Their approach makes use of a batterycentric objective function which requires a precise knowledge of the battery level. This information is not always realistic or accurate and it is better use it in a complementary manner [13]. Another contribution of their work is the consideration of duty-cycle stability, the issue addressed for the first time. The authors change the duty-cycle using a smoothing system to reduce the duty-cycle variability. The approach may be a good solution for wireless sensor networks in general, but not for the IEEE 802.15.4 beacon-enabled mode in which the dutycycle is bounded to specific values of BO and SO.

Yoo et al. evaluated two adaptive duty-cycle schemes in a harvesting and multi-hop context [1]. The first algorithm computes its duty-cycle in an inverse proportion to the battery residual energy. The second one also benefits from an estimation of the residual energy increase allowing for more aggressiveness. However, this evaluation is based on an asynchronous duty-cycle protocol (when 802.15 .4 belongs to synchronous ones) and does not consider dynamic harvesting profiles.

With respect to the related work, our adaptation scheme presents the advantage of taking into account the general multi-hop topology of the network as well as the complex interaction between the BO and SO values of parent nodes and possible values of the $\mathrm{BO}$ and $\mathrm{SO}$ at a given node.

\section{CONCLUSION}

In the present paper, we propose a novel adaptation scheme that takes into account the energy harvesting rate, the battery level and local traffic conditions. It provides the best quality of service for the available level of energy in an IEEE 802.15.4 beacon-enabled multi-hop harvested WSN. The scheme uses an analogy of the token bucket applied to the harvested intake energy, the model we call an energy bucket. The adaptation scheme takes into account the intake energy, stored energy, the buffer status of son nodes, and finds the best duty-cycle that leads to high throughput and low delay. It also reserves some energy for surviving the periods without energy intake, and energy to face eventual buffer queuing avoiding packet drop.

Our evaluation through simulations has shown that the proposed scheme leads to good performance in terms of packet delay and packet delivery ratio as well as to an efficient use of harvested energy. As a future direction of this work, we plan to deploy a network of STM solar harvested nodes with the adaptation scheme and measure its performance in real conditions. Moreover we are interested in studying how to dynamically adapt $\alpha, \beta$ and $\gamma$ to different network situations (traffic and light pattern). This experience will also help us to empirically characterize the battery behavior and charging times of the STMicroelectronics sensor node in time and space.

\section{REFERENCES}

[1] H. Yoo, M. Shim, and D. Kim, "Dynamic duty-cycle scheduling schemes for energy-harvesting wireless sensor networks," Communications Letters, IEEE, vol. 16, no. 2, pp. 202-204, 2012.

[2] IEEE Std 802.15.4-2011 (Revision of IEEE Std 802.15.4-2006), 2011.

[3] (2013, dec) An event-driven simulator for large wireless sensor networks. [Online]. Available: http://wsnet.gforge.inria.fr/index.html

[4] N. Abdeddaim and F. Theoleyre, "Implementation of a wsnet module to simulate the ieee 802.15 .4 beacon-enabled mode in multihop topologies," HAL, Tech. Rep. 00590853, May 2011, http://hal.archivesouvertes.fr/hal-00590853/.

[5] Y. Sun, O. Gurewitz, and D. B. Johnson, "RI-MAC: A receiver-initiated asynchronous duty cycle mac protocol for dynamic traffic loads in wireless sensor networks," in Proc. of ACM SenSys'08, 2008.

[6] "Hitachi Maxell lithium manganese dioxide rechargeable battery - technical report," http://biz.maxell.com/files_etc/6/catalog/en/ML_13e.pdf, Sep. 2014.

[7] M. Gorlatova, M. Zapas, E. Xu, M. Bahlke, I. J. Kymissis, and G. Zussman, "CRAWDAD data set columbia/enhants (v. 2011-04-07)," Downloaded from http://crawdad.org/columbia/enhants/, Oct. 2014.

[8] R. de Paz Alberola and D. Pesch, "Duty cycle learning algorithm (DCLA) for ieee 802.15.4 beacon-enabled wireless sensor networks," Ad Hoc Networks, vol. 10, no. 4, pp. 664-679, 2012.

[9] R. de Paz Alberola, B. Villaverde, and D. Pesch, "Distributed duty cycle management (DDCM) for ieee 802.15.4 beacon-enabled wireless mesh sensor networks," in Mobile Adhoc and Sensor Systems (MASS), 2011 IEEE 8th International Conference on, Oct 2011, pp. 721-726.

[10] IEEE Std 802.15.4e-2012 (Amendment to IEEE Std 802.15.4-2011), 2012.

[11] A. Kansal, J. Hsu, S. Zahedi, and M. B. Srivastava, "Power management in energy harvesting sensor networks," ACM Transactions on Embedded Computing Systems (TECS), vol. 6, no. 4, p. 32, 2007.

[12] C. M. Vigorito, D. Ganesan, and A. G. Barto, "Adaptive control of duty cycling in energy-harvesting wireless sensor networks," in Proc. of IEEE SECON'07, 2007.

[13] N. Michelusi, L. Badia, R. Carli, K. Stamatiou, and M. Zorzi, "Correlated energy generation and imperfect state-of-charge knowledge in energy harvesting devices," in Wireless Communications and Mobile Computing Conference (IWCMC), 2012 8th International. IEEE, 2012, pp. 401-406. 